\title{
Humoral autoreactivity to an alternatively spliced variant of ICA512/IA-2 in Type I diabetes
}

\author{
Y.S. Park ${ }^{1}$, E. Kawasaki ${ }^{2}$, K. Kelemen ${ }^{1}$, L. Yu ${ }^{1}$, M. R.Schiller ${ }^{3}$, M. Rewers ${ }^{4}$, M.Mizuta ${ }^{5}$, G. S. Eisenbarth ${ }^{1}$, \\ J.C.Hutton ${ }^{1}$ \\ ${ }^{1}$ Barbara Davis Center for Childhood Diabetes, University of Colorado Health Sciences Center, Denver, USA \\ ${ }^{2}$ The First Department of Internal Medicine, Nagasaki University School of Medicine, Nagasaki, Japan \\ ${ }^{3}$ Department of Pathology and Anesthesiology/Critical Care Medicine, Johns Hopkins University, Baltimore, USA \\ ${ }^{4}$ Department of Preventive Medicine and Biometrics, University of Colorado, School of Medicine, Denver, USA \\ ${ }^{5}$ The Third Department of Internal Medicine, Miyazaki Medical College, Miyazaki, Japan
}

\section{Abstract}

Aims/hypothesis. The receptor tyrosine phosphatase like-protein ICA512/IA-2 occurs as a proteolyticallyprocessed $65,000 \mathrm{M}_{\mathrm{r}}$ type 1 transmembrane glycoprotein in beta cells and is a major autoantigen of Type I (insulin-dependent) diabetes mellitus. We investigated whether alternative splicing could affect humoral autoreactivity to the molecule.

Methods. Genomic and cDNA sequence analysis showed the presence of a ICA512 variant in islets and lymphoid tissues with an in-frame deletion of exon 13 which produces a secreted form lacking aa 557-629 including the transmembrane domain (aa 577 to 600 ). The alternatively spliced protein is detectable by western blotting in normal islets and translated into a protein that is processed to a series of soluble forms of 25,000-35,000 $\mathrm{M}_{\mathrm{r}}$. Radioimmunoprecipitation assays for anti-ICA512 autoantibodies were developed with the widely used ICA512.bdc construct (which has exon 13 deleted) and a series of full-length and modified ICA512/IA-2 molecules.
Results. The assays showed that ICA512.bdc and ICA512 $604-979$ gave the best discrimination between diabetic and control sera. With ICA512 ${ }_{604-979}$ a somewhat greater proportion of patients expressing antibodies were detected than with ICA512.bdc in the groups studied $(70.5 \%$ vs $63.2 \%$ of prediabetic/newonset and 25.0 vs $13.9 \%$ in patients with diabetes $>20$ years). Conversely, a small proportion ( $3 \%$ recent-onset and $6 \%>20$ years) had antibodies to ICA512.bdc but not ICA512 $604-979$.

Conclusion/interpretation. Important epitopes lie within the exon 13 region and others can be generated by the alternative splicing. As the $\Delta$ exon 13 variant is probably secreted by the beta cell, it could be recognized by the cellular and humoral arm of the immune system in the absence of cellular damage. [Diabetologia (2000) 43: 1293-1301]

Keywords Insulin-dependent diabetes mellitus, alternative splicing variant, ICA512/IA-2.
A number of autoantigens have been identified in Type I (insulin-dependent) diabetes mellitus, including insulin [1], glutamic acid decarboxylase (GAD)

Received: 13 March 2000 and in revised form: 5 June 2000

Corresponding author: J.C. Hutton, PhD, Barbara Davis Center for Childhood Diabetes, University of Colorado Health Sciences Center Box B-140, 4200 E. $9^{\text {th }}$ Avenue, Denver CO 80262, USA

Abbreviations: PTP, Protein tyrosine phosphatase; JM, juxta membrane; TM, transmembrane; RIPA, radioimmunoprecipitation assay.
[2-6], carboxypepetidase $\mathrm{H}$ [7], the glycolipid GM2-1 [8], islet cell autoantigen 69 [9] and the protein tyrosine phosphatase (PTP)-like proteins ICA512 [10-14] and phogrin (IA-2 $\beta$ ) $[15,16]$. Autoantibodies (AA) as such have yet to be shown to play a major part in the pathogenesis of the disease and could represent "epiphenomena" of a beta-cell attack. Nevertheless, they provide valuable markers to predict Type I diabetes and confirm the diagnosis of autoimmunity [17-20].

The combined measurement of ICA512, GAD65 and insulin autoantibodies is the most reliable marker for the diagnosis and prediction of Type I diabetes. 
We initially cloned ICA512 by screening a human islet cDNA expression library using a pool of sera from patients with Type I diabetes [10, 21] and IA-2 from a subtractive cDNA library [12]. Discrepancies between the sequences of IA-2 and ICA512 were subsequently attributed to sequencing errors and it was resolved that both cDNAs encode a 979-amino acid protein with a molecular mass of $106,000 \mathrm{M}_{\mathrm{r}}$, with a single transmembrane (TM) region (aa 577-600). The protein is found in cells of the diffuse neuroendocrine system within the membranes of secretory granules as a proteolytically-processed 65,000 $\mathrm{M}_{\mathrm{r}}$ form (aa 418-979) [22].

Our initial investigations of humoral autoimmunity to ICA512 used a cloned partial sequence (clone HB-1; nt 837-3613) obtained from a human islet cDNA expression library. We, and others, have developed radioimmunoprecipitation assays (RIP) for anti-ICA512 using in vitro transcribed and translated products derived from the HB-1 sequence subcloned into pGEM vector (designated ICA512.bdc hereafter). The predominant epitopes of ICA512 have been localized in its cytoplasmic segment which incorporated both the PTP domain (amino acids 687-979) and the juxta-membrane (JM) amino acids 601-686 [14, 23, 24]. We report here that both the ICA512.bdc sequence and the parental clone (HB-1) lack the TM domain of the protein as a result of alternative splicing that deletes exon 13 (aa 577-629) [25]. Because the JM region incorporates important immunological epitopes and an alternatively spliced variant could contain unique conformational epitopes, we have investigated a series of in vitro produced ICA512 variants as targets of Type I diabetesassociated autoantibodies.

\section{Subjects and methods}

Unless otherwise stated, all molecular cloning procedures were done by standard protocols and reagents were of analytical grade and purchased from Sigma (St. Louis, Mo., USA).

Nomenclature. The term ICA512 is used to designate the product of the ICA512/IA-2 gene. ICA512 and IA-2 refer to the same gene product. ICA512bdc refers to a specific cDNA clone used extensively in assays of autoreactivity in Type I diabetes. It is truncated $\mathrm{NH} 2$ terminally and carries a deletion of exon 13 and thus can be designated IA2/ICA512 ${ }_{256-556630-979}$.

Islets, tissues and cell lines. Islets were isolated from 5 to 7-dayold newborn Sprague-Dawley rats and $B A L B / C$ mice as described previously [26]. Human islets were isolated from two human cadaveric donors (gift from Dr. Camillo Ricordi, University of Miami, Medical School) by collagenase digestion as described previously [27]. Rat pituitary, pancreatic islets, brains, thymus, spleen and adrenal tissues were also isolated, homogenized and digested into single cells. The mouse and rat insulinoma cell lines, $\beta \mathrm{TC} 3$, MIN6, and INS cells were maintained in tissue culture in Dulbecco's Modified Eagle's
Medium (DMEM) or RPMI 1640 medium (Gibco BRL, Paisley, UK) containing $10 \%$ FCS (Sigma Chemical Co., Poole, Dorset, UK) until they were subconfluent.

RT-PCR analysis. We extracted RNA from the above tissues using Trizol reagent (Life Technologies, Long Island, N.Y., USA) and isolated Poly(A) + mRNA using oligo(dT)-cellulose (Life Technologies) following the manufacturer's instructions. We generated cDNA from total RNA by reverse transcription and then subjected it to PCR amplification (RTPCR) run for 35 cycles in a Thermoline thermocycler (1 min at $94^{\circ} \mathrm{C}, 1 \mathrm{~min}$ at $55^{\circ} \mathrm{C}, 2 \mathrm{~min}$ at $72^{\circ} \mathrm{C}$ ). The PCR products were visualized on ethidium bromide stained $2 \%$ agarose gels. The primers IA-2-F 5'-AGA CAG GGC TCC AAA TCT TGC-3' and IA-2-R 5'-GCC TGG GCT GCG TCG CTG AAC-3' were used to amplify both the full length IA-2 mRNA ( 370 bp) and the alternatively spliced transcript lacking exon 13 (151 bp). Parallel reactions using template without reverse transcriptase enzyme were run to control for genomic DNA contamination. The quality of the RNA was checked in all tissues studied by evaluating the expression of $\beta$-actin mRNA.

Sequencing and Southern blot analysis of human genomic $D N A$. Genomic DNA was isolated from a healthy person and nucleotide sequence spanning exon 13 and the flanking introns was analysed in both directions with a ABI 373A sequencer (Applied Biosystems, Foster City, Calif., USA) using primers 5'-AGACAGGGCTCCAAATCTTGC-3' and 5'GCCTGGGCTGCGTCGCTGAAC-3' corresponding to 1704-1724 and 2053-2033, of ICA512 cDNA together with internal primers. Southern blotting of genomic DNA was done after digestion with Apa I, Sph I and Nco I. The probe was derived from the intracellular portion of ICA512 cDNA (974 bp, nt 2124-3097, aa 655-979) and labelled with digoxigenin (DIG, Boehringer Mannheim, Mannheim, Germany) according to the manufacturer's instructions.

Patients and sera. Sera were obtained from 121 healthy control subjects with no family history of diabetes, 279 subjects with Type I diabetes from the Joslin Diabetes Center and the Barbara Davis Center. The latter were comprised of 51 (27 male and 24 female) relatives of patients with Type I diabetes followed to onset of disease, 71 (36 male and 35 female) new-onset Type I diabetic patients tested within 7 days of diagnosis, 34 (18 male and 16 female) recent-onset Type I diabetic patients (duration $<1$ year), 52 (25 male and 27 female) intermediate-duration diabetic patients (duration 1-10 years), and 71 patients with long-duration diabetes (duration $>10$ years). The median age of the patient groups at sampling was 10.4 years (range: 1.1-37.4 years), 9.2 years (range: $0.4-41.5$ years), 10.4 years (range 1.2-51.7 years), 16.0 years (range 2.5-43.5 years) and 30.2 years (range: 11.3-77.8 years), respectively. The median age of control subjects was 20.2 years (range: 7.1 to 51.4 years). Sequential sera samples were also obtained from 50 subjects of the DAISY (Diabetes Autoimmune Study in the Young) cohort who were followed from the finding of autoantibody positivity (GAD, ICA512.bdc or IAA) to overt diabetes [28]. Of these 40 were siblings or offspring of patients with Type I diabetes and 10 were newborns from the general population. The median age of relatives at initial assay was 2.8 years (range $0-8.0$ years) and that of the non-relative newborns was 0.8 year (range 0-2.7 years). The mean follow-up of the former was $2.0 \pm 1.6$ years and the latter was $1.4 \pm 1.1$ years. All sera were stored at $-20^{\circ} \mathrm{C}$ until use. Diagnosis of Type I diabetes accorded with the criteria of the ADA commission [29]. All subjects gave informed consent and protocols were approved by the Institutional Review Boards of the University of Colorado. 


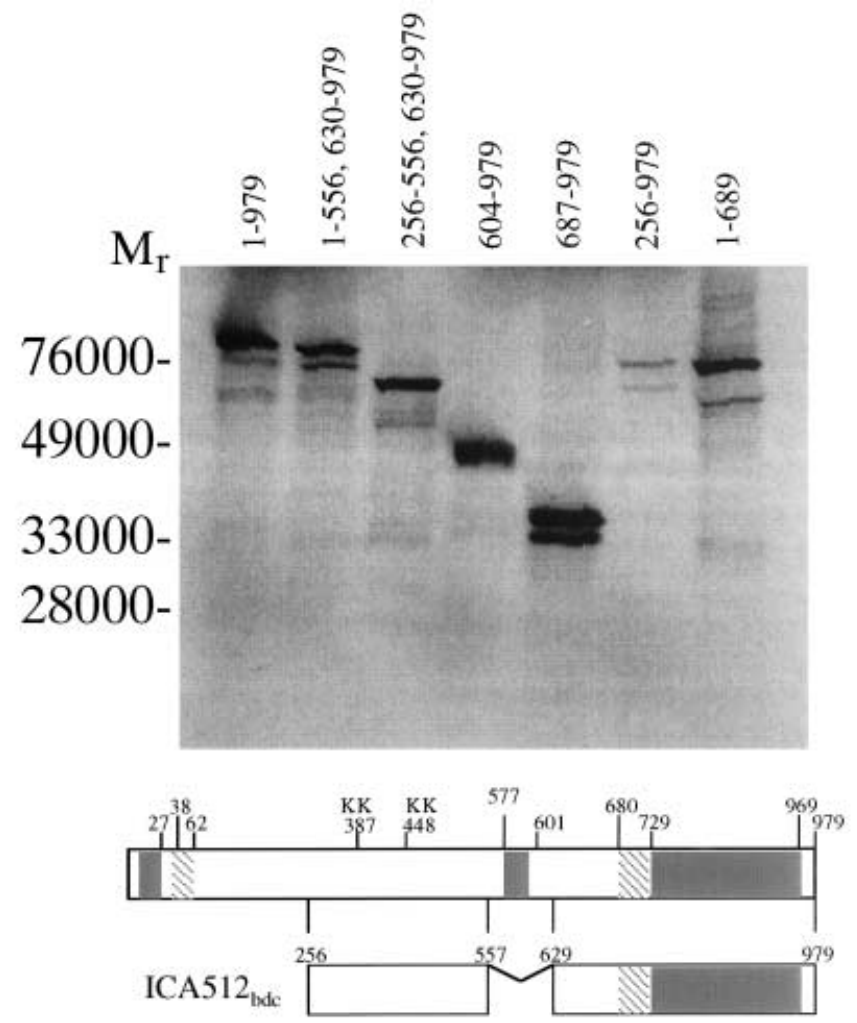

Fig. 1. In vitro translation of ICA512 constructs. A schematic representation of the full-length ICA512 and $\Delta$ exon 13 variant is shown. The signal sequence, cysteine-rich domain, transmembrane domain and PTP domain are shaded. The hatched region preceding the $\mathrm{PTP}$ domain is conserved between ICA512 and phogrin but not other PTP members

cDNA cloning. We (Fig. 1) generated seven constructs of the ICA512 molecule by PCR using Taq polymerase (Ampli Taq, PE Biosystems, Foster City, Calif., USA) from the full-length ICA512 cDNA (ICA512 1-979) provided by Dr. E. Bonifacio (University of Milan, Italy) or the partial clone (nt 837-3613 encoding aa 389-948) described previously (Table 1) [11, 30]. Constructs were generated by PCR of cDNA sequence spanning the start codon to the PTP domain (ICA512 $11_{-689}$ ), the PTP domain alone (ICA512 $687-979$ ), the complete cytoplasmic region (ICA512 $604-979$ ), ICA512.bdc (ICA512 256-979; $4577-629$ ), and "full length" $\triangle$ exon 13 form deletion (ICA 512 $1-979$; ${ }_{\Delta 577-629}$ ). A Kozak consensus start codon was built into the primer sequence as needed and the constructs cloned directly into the pCRII vector (Invitrogen, San Diego, Calif., USA) or pGEM-T vector (Promega, Madison, Wis., USA). The sense and antisense primers for the deletion constructs we made were as follows.
ICA512 $1-689$
5'-GAGTTCGGAAAGATGCGG-3'
ICA512 $687-979$
5'-AAGCCAACATGGACATCT-3'
ICA512 $604-979$
侐
5'-GGTACACAGAGAGATGCTCA-3'
ICA512 $256-979$
5'-ACCATGGCGCGGCAGCAAGA-3'
5'-GGTACACAGAGAGATGCTCA-3'
5'-ACATGCCTGGCCACTCCTA-3'
5'-GGTACACAGAGAGATGCTCA-3'
ICA512 $1-979$

The nucleotide sequence of each construct was confirmed by automated sequencing in both directions. The aminoacid numbering is based on the deposited amino-acid sequence of human ICA512 (Gene Bank accession number L18983).

Western blotting. Groups of 1600 human islets were homogenized in $100 \mu \mathrm{l}$ of PBS containing $2 \mu \mathrm{mol} / \mathrm{l}$ Pepstatin A, $10 \mu \mathrm{mol} / 1 \mathrm{E}-64$ and $0.1 \mathrm{mmol} / \mathrm{l}$ PMSF. Homogenates were centrifuged for $2 \mathrm{~h}$ at 259,000 $\mathrm{g}$ in a Beckman TLS 55 rotor. Samples $(100 \mu \mathrm{g}$ protein) of the crude homogenate, the soluble fraction (supernatant) and the resuspended membrane fraction (pellet) were electrophoresed on $12.5 \%$ SDS-PAGE gels, transferred to nitrocellulose and immunoblotted with rabbit antibodies (1:500) generated to the lumenal domain of the mature protein (aa 389-576: Fig. 1). Blots were developed with an alkaline phosphatase-conjugated secondary antibody using a proprietary fluorescent substrate (Vistra ECF reagent; Amersham International, Amersham, Bucks, UK) and quantified by fluorescence imaging (Storm 860; Molecular Dynamics, Sunnyvale, Calif., USA).

Autoantibody radioimmunoprecipitation assays (RIPA). The cloned constructs were transcribed and translated in vitro in the presence of ${ }^{35}$ S-methionine (Amersham International; > 37 $\mathrm{TBq} / \mathrm{mmol}$ ) using the TNT-coupled rabbit reticulocyte system (Promega) (Fig. 1). Radioimmunoprecipitation assays were done in a 96-well plate format as described previously [30]. In brief, ${ }^{35}$ S-labelled protein $(20,000 \mathrm{cpm})$ was incubated with patients' sera at a $1: 25$ dilution overnight at $4{ }^{\circ} \mathrm{C}$ in $100 \mu \mathrm{l}$ TBST buffer (20 mmol/1 TRIS-HCl, pH 7.4, 150 mmol/1 NaCl, 0.1\% bovine serum albumin, $0.15 \%$ Tween 20 containing $0.1 \%$ aprotinin and $10 \mathrm{mmol} / \mathrm{l}$ benzamidine). Samples $(50 \mu \mathrm{l})$ were transferred to MultiScreen-DV 96-well filtration plate (Millipore, Burlington, Mass., USA) and $25 \mu \mathrm{l}$ of $50 \%$ (w/v) Protein A-Sepharose (Pharmacia, Uppsala, Sweden) in TBST added. After incubation for $45 \mathrm{~min}$ at $4^{\circ} \mathrm{C}$, immunoprecipitates were washed nine times with ice-cold TBST using the Millipore vacuum-operated 96-well plate washer (Millipore, Bedford,

Table 1. Comparison of $99 \%$ index, control mean index, SD score and intra-assay and inter-assay variances between different constructs

\begin{tabular}{|c|c|c|c|c|c|}
\hline Construct & $99 \%$ Index & Control mean index & Diabetes SD score & Inter-assay & Intra-assay \\
\hline$(1-979)$ & 0.111 & $0.004 \pm 0.037$ & $7.2 \pm 0.8$ & $6.5(9)$ & $10.1(10)$ \\
\hline$(1-556,630-979)$ & 0.319 & $-0.011 \pm 0.0157$ & $2.4 \pm 0.2$ & $11.2(8)$ & $10.2(9)$ \\
\hline$(256-556,630-979)$ & 0.049 & $0.001 \pm 0.010$ & $59.5 \pm 5.4$ & $11.7(9)$ & $10.0(9)$ \\
\hline (687-979) & 0.031 & $-0.001 \pm 0.010$ & $31.6 \pm 3.1$ & $9.9(9)$ & $10.0(10)$ \\
\hline (256-979) & 0.000 & $-0.005 \pm 0.075$ & $4.5 \pm 1.0$ & $9.2(8)$ & $10.0(10)$ \\
\hline
\end{tabular}




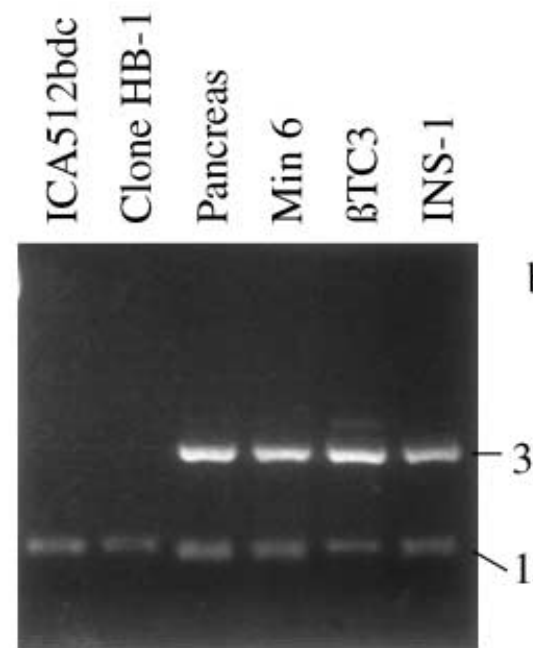

Fig. 2. Expression of the ICA512 mRNA and its variant in different tissues and beta-cell lines (MIN6, $\beta$ TC3 and INS- 1 cells respectively). The ICA512 cDNA (370 bp) and the $\Delta$ exon 13 variant (151 bp) were amplified from the cDNAs by PCR using the primers spanning the deleted region

Mass., USA). Then, $40 \mu$ of scintillation liquid (Microscint-20; Packard, Meriden, Conn., USA) was added to each well and radioactivity measured with a Top Count 96-well plate scintillation counter (Packard). Positive and negative control sera were included in every assay and the antibody binding expressed as an index defined as: (unknown sample cpm-negative control cpm)/(positive control cpm-negative control cpm). "Positive" was based on the 99th centile of sera from 121 healthy control subjects.

Competition studies. The cDNA encoding ICA512 ${ }_{1-689}$ and ICA512 $687-979$ were expressed as glutathione S-transferase (GST) fusion proteins in XL-1 blue E.coli using the pGEX $3 \mathrm{X}$ vector (Pharmacia, Arlington Heights, Ill., USA). Recombinant protein expression was induced with $0.1 \mathrm{mmol} / \mathrm{l}$ IPTG (isopropyl- $\beta$-D-thiogalactopyranoside) and the soluble product purified by glutathione-Sepharose affinity chromatography. The GST portion of each molecule was cleaved by factor Xa treatment and the GST removed by a further round of glutathione-Sepharose affinity chromatography [31]. The homogeneity of the recombinant protein was confirmed on $12 \%$ SDS-PAGE with Coomassie blue staining. Competition studies were carried out by pre-absorption of sera (1:10 dilution) in $100 \mu \mathrm{l} \mathrm{TBST}$ with $50 \mu \mathrm{g}$ of the purified recombinant proteins for $3 \mathrm{~h}$ at room temperature. We then mixed $40 \mu \mathrm{l}$ of the sample with in vitro translated ${ }^{35} \mathrm{~S}$ labelled antigen $(20,000 \mathrm{cpm}$ of TCA precipitable protein) in $100 \mu \mathrm{l}$ TBST (final volume). Samples were then processed in the autoantibody immunoprecipitation assay described above.

Statistical analysis. Differences between the autoantibody prevalence for the patients with different duration of diabetes were calculated by the $\varkappa^{2}$ test with Yates' correction (twotailed). When the expected cell value in a $2 \times 2$ table was less than 5, the Fisher's exact test was used. A $p$ value less than 0.05 was considered statistically significant.

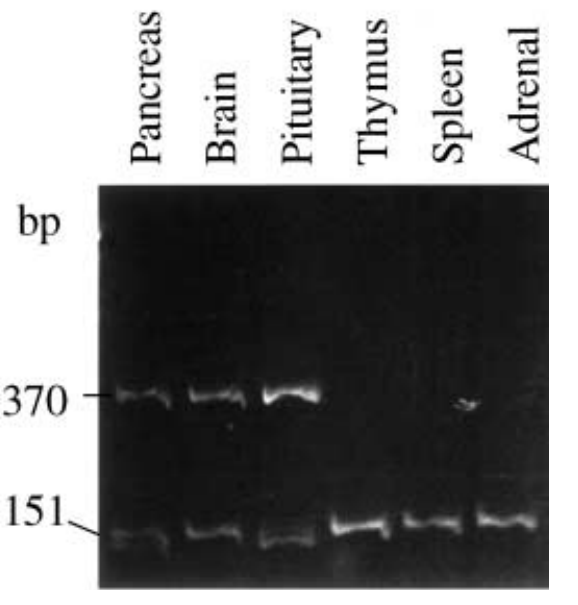

\section{Results}

Sequence analysis of ICA512.bdc. Full-length DNA sequence analysis of ICA512.bdc showed that residues 1742 to 1960 of ICA512 sequence were absent, a deletion which would result in an in-frame deletion of 73 aa [aa 557-629 a region that includes the TM domain (aa 577 to 600)]. We confirmed the deletion of the same region in the original cDNA subclone (HB-1) from which ICA512.bdc was derived. The encoded protein is 906 aa in length with a molecular mass of $98,000 \mathrm{M}_{\mathrm{r}}$

Reverse transcriptase polymerase chain reaction ( $R T$ $P C R$ ). We did RT-PCR analysis of mRNA from islet

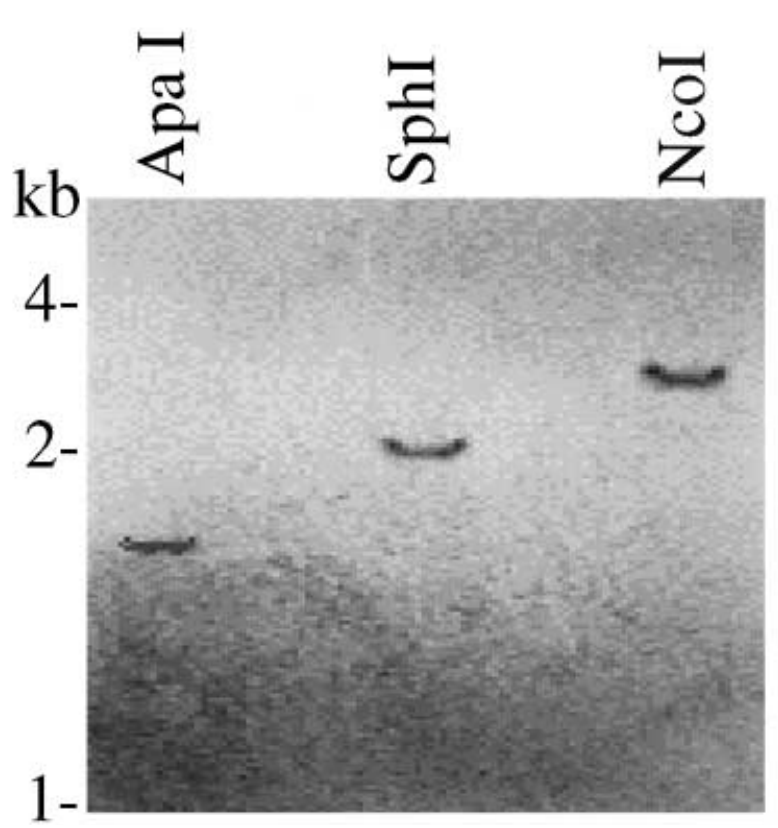

Fig.3. Southern blotting of the gene for ICA512 in human genomic DNA. A 974 bp probe encoding intracellular ICA512 cDNA was labelled by random priming with digoxigenin 


\begin{abstract}
CAGACAGGGCTCCAAATCTTGCAGACAGGAGTGGGACAGgtatgcgctaactggagaatcagtcC aagtccccagagggetgatgttgtccgcaagagctggaggcaaaccagaggggagaactggagcC gaaacgtgtccgtaggcacggetggetggaggcggecttcgcccaagcagcccctccccgcagtt ctctttctcaccagcagatggcgtaggtgtcccctccctgcaggaccagccctctgtagtaaaaa ggcctgagaactagttgagccgcccttagtctgctggagcgcagtctccaaattccaagggaaca ccttgcacagaaaggacattatactcaaaatatgttttgtttgtttgtctgagacagagtttcac tcttgctgttccagctggagtgcagtggctcgatctcggctcactgcaacctccacctgccaggt tcaagcgattctcctgcctcagcctcccaagtagctgggattacaggtgcctgccaccacacceg ggtaaattttttttgtatttttagtagagatggggtttcaccatgttggccaggctggtctcga actcccgatctcaggtgatccgccaacctttggccccccaaagtgctgagattacaggtgtgagc caccacgcccggcctatactcaaaatacctttgtcttctgcatccaacactgaatgcctcacgtt ctacaattcaattcctagggttctctgtccgtttttaaatgcaaacactccctggtgggggcctc tggcactgcactgtggttgagttgatccgatggggattgagaaggagaatctgagccatctggc tgtaccaccatacccctaatctcccagctttgccccaggagatgtcctaacagggggtgtggcat gggaccggggtgaggagcagctctgaaacctccctatgccactgcctcccttgattctagccttg ttcccacagAGGGAGGAGGCAGCTGCAGTCCTTCCCCAAACTGCGCACAGCACCTCACCCATGCG CTCAGTGCTGCTCACTCTGGTGGCCCTGGCAGGTGTGGCTGGGCTGCTGGTGGCTCTGGCTGTGG CTCTGTGTGTGCGGCAGCATGCGCGGCAGCAAGACAAGGAGCGCCTGGCAGCCCTGGGGCCTGAG GGGGCCCATGGTGACACTACCTTTGAGTACCAGgtgtgcagccccagaagctgattgagcaaagg aaatgcaaggggcctgggagtctgggtcttgcgtggactaagggtcggaactgaatggggagtga gccctcatccctgggaaggtccagtggaggctggagaagccccagtgggagggagtatggtaggg gccttatgaacgcatgggaattgggagcatacagtgcctaaggttcttcttggtgctgtgcttc tggggctctgggattccactctgtcatgtctgaggttgacataagcaccacccaagcaaaacagc cctggaaaggatcagggccaggcagaagaccataccccagaggagctgagttccactgaaaatca gtcaagggcaagcctaggagcccagagttgaacccagggccctgttgaaaagtttcaggggactt gGACCTCACCTGATTCTTCCTCCACAGGACCTGTGCCGCCAGC
\end{abstract}

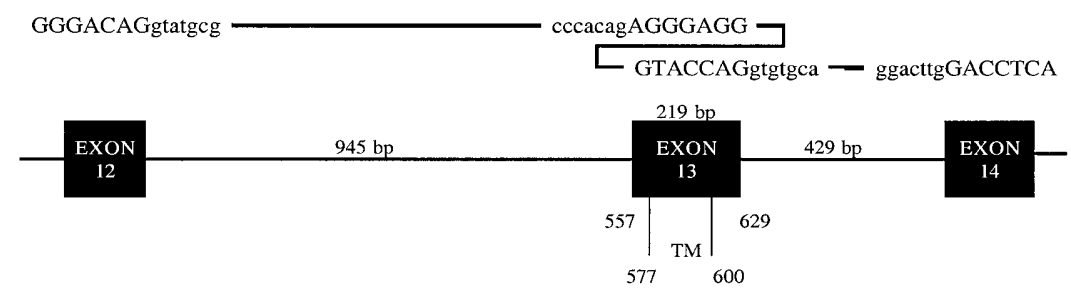

Fig.4. Nucleotide sequence of human genomic DNA around the deleted region. Intronic sequence appears in lower case

cells using primers flanking the deleted region ( $\mathrm{P} 1$ and $\mathrm{P} 2$ ). Two bands were amplified from human islets as well as rodent islet beta-cell lines Min 6, $\beta \mathrm{TC} 3$ and INS-1 (Fig. 2). The smaller PCR product corresponded in size to the single band amplified from the ICA512.bdc clone and the original lambda clone HB-1. Sequencing of the PCR products confirmed that the bands corresponded to the deleted (151 bp) and non-deleted (370 bp) forms, respectively (data not shown). Bands of similar size were amplified from cDNA derived from the rat islets, adrenal, pituitary, brain, thymus and spleen tissue (Fig. 2).

Southern blot analysis and sequencing of human genomic DNA. A cDNA fragment of ICA512 cDNA (974 bp, nt 2124-3097, aa 655-979) which includes exon 14 was used to probe the gene in human genomic DNA digested with different enzymes which were predicted to cut once within the deleted transmembrane region. In every case a single hybridizing sequence was observed indicating that the $\Delta$ exon 13 variant arose from alternative splicing rather than being the product of an allelic variant or separate gene (Fig. 3). The human genomic sequence contained the 219 bp-deleted region (exon 13) flanked by 945 bp and $429 \mathrm{bp}$ of intronic sequences (Fig. 4).
Western blotting analysis. Western blot analysis of human islet homogenates showed the presence of the major $65,000 \mathrm{M}_{\mathrm{r}}$ protein along with some minor components of lower molecular size $\left(48,000 \mathrm{M}_{\mathrm{r}}, 30,000 \mathrm{M}_{\mathrm{r}}\right.$ and 25,000 $\mathrm{M}_{\mathrm{r}}$ ) (Fig.5). None of the bands were detectable after pre-absorption of the serum with recombinant GST-full-length ICA512 molecule. Fractionation of the islet tissue showed that the 65,000 $\mathrm{M}_{\mathrm{r}}$ component was pelleted and could be solubilized with $0.1 \%$ Triton X-100 (not shown) consistent with its known localization in dense-core granule membranes. The $48,000 \mathrm{M}_{\mathrm{r}}$ component behaved in a similar manner, the broadly migrating bands in the 25,000-33,000 $\mathrm{M}_{\mathrm{r}}$ range were, however, released into the supernatant after homogenization. The $\Delta$ exon 13 mutant form of ICA512, if translated, would be expected to be produced initially as a soluble lumenal protein of around $100,000 \mathrm{M}_{\mathrm{r}}$ and subsequently converted to a $50,000-55,000 \mathrm{M}_{\mathrm{r}}$ form if glycated and processed at the KK448 site. The soluble forms observed were clearly shorter presumably as a result of further proteolytic degradation. Western blotting analyses with a cytosolic domain antibody were uninformative principally because of its cross-reactivity with phogrin (IA-2 $\beta$ ). Densitometric analysis on two separate human islet preparation suggested that the $25,000-33,000 \mathrm{M}_{\mathrm{r}}$ forms comprised around 10-15\% of the $65,000 \mathrm{M}_{\mathrm{r}}$ immunoreactivity on a molar basis.

Autoantibody RIPA. The ICA512.bdc and an additional six ICA512 mutant constructs were produced 


\section{Hom. SN Pell.}

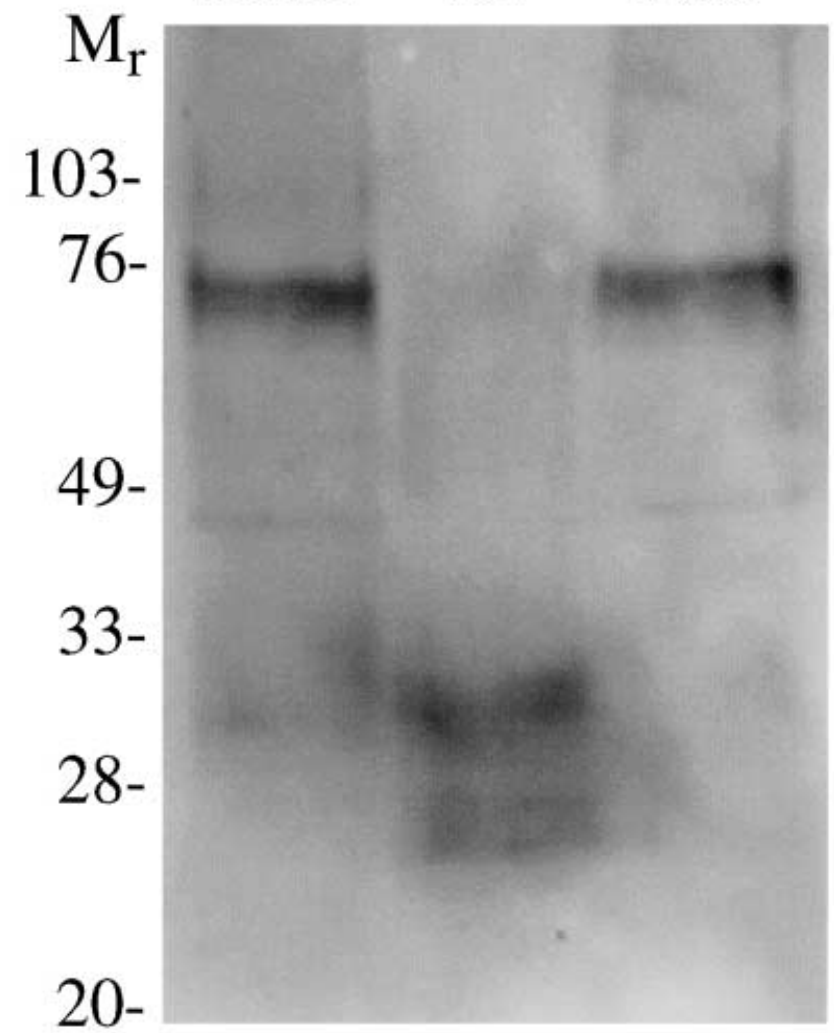

Fig. 5. Western blot analysis of fractionated human islet tissue. The crude homogenate (Hom.) was centrifuged at 290,000 $\times g$ to generate a soluble (SN) and pellet (Pell). The gel loading of the Pell fraction was adjusted to an equivalent amount of membrane protein as Hom. SN was loaded to an equivalent of fivefold the amount of starting material

using the rabbit reticulocyte lysate in vitro translation system. The SDS-PAGE analysis confirmed the predicted sizes of the translation product (Fig.1) although some heterogeneity existed possibly as the result of alternative AUG start codons in the molecule.

Sera were considered autoantibody positive when the radioactivity precipitated exceeded the $99^{\text {th }}$ centile of normal control sera. The assay threshold index and the mean control index for each construct showed considerable variation even though the intra-assay and inter-assay covariance were generally in the same range (Table 1). This was related to background cpm observed in the assays with each construct and seemed to be a function of the extent of incorporation of the lumenal domain sequence, a region which does not contain diabetes-related epitopes. The incorporation of the hydrophobic transmembrane domain did not seem to be a factor.

Of the prediabetic and new-onset Type I diabetic patients $70 \%$ (86 of 122), $64 \%$ (78 of 122$), 63 \%(77$ of 122 ), $70 \%$ (86 of 122$), 57 \%$ (70 of 122 ) and $61 \%$ (75 of 122) had autoantibody levels exceeding the $99^{\text {th }}$ centile of the healthy control group to

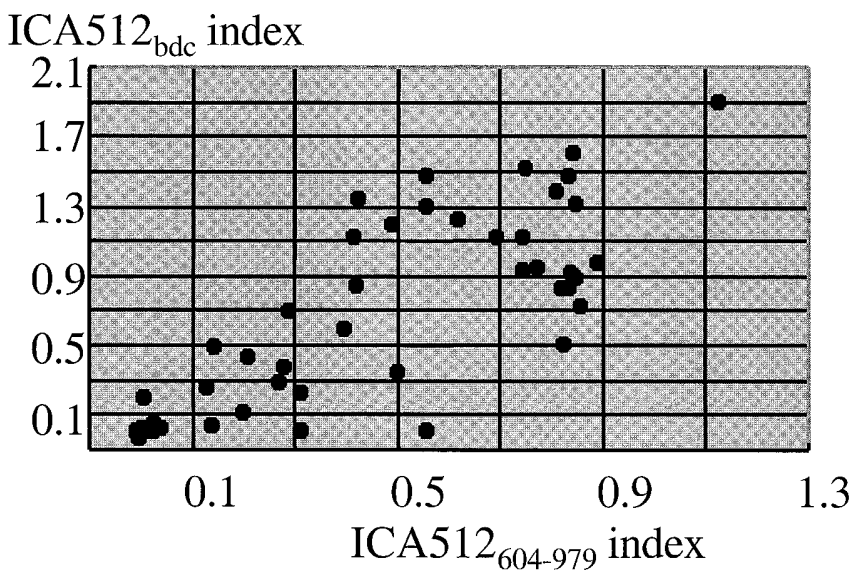

Fig. 6. Correlation of autoantibody titres obtained from diabetic sera using ICA512 $2_{\mathrm{bdc}}$ and ICA512 $604-979$. The cut-off index in each assay corresponds to 0.1 in each case

ICA512 $1-979, \quad$ ICA512 $1-556, \quad{ }_{630-979,}$ ICA512.bdc, ICA512 $604-979$, ICA512 $687-979$ and ICA512 $256-979$, respectively. Of patients' sera $25 \%$ were negative for all of the constructs and $21 \%$ of them were positive for all of the constructs. Direct quantitative comparison of the different assays was possible only where the $99^{\text {th }}$ centile index and control mean index were similar and was thus restricted to assays using ICA512bdc, ICA512 ${ }_{604-979}$, and ICA512 ${ }_{687-979}$. (Table 1). The ICA512bdc, ICA512 $604-979$ and ICA512 $687-979$ gave notably better discrimination between diabetic and control sera with a higher mean SD score compared with the other constructs $(p<0.05)$. The titre of autoantibodies detected by these assays showed broad correlation as illustrated in Figure 6 for ICA512bdc compared with ICA512 $604-979$.

All new-onset Type I diabetic patients positive for the ICA512 ${ }_{687-979}$ construct were also positive for ICA512 ${ }_{604-979}$ and ICA512.bdc $256-556,630-979$ in accordance with their common possession of the PTP domain. In prediabetic and recent-onset patients (duration $<1$ year $), 18(11.5 \%, 18 / 156)$ had autoantibodies to ICA512 $604-979$ (Table 2), without reactivity to ICA512.bdc suggesting that some epitopes are dependent on the preservation of the region between aa 604-629. Conversely 5/156 (3.2\%) had autoantibodies to ICA512.bdc, but not to the ICA512 $604-979$. With increasing duration of diabetes, there was 10 years duration group 10/71 (14.1\%,) had autoantibodies to ICA512 $604-979$, but not to the ICA512.bdc $256-556,630-979$ compared with $4 / 71$ (6\%) with autoantibodies to ICA512.bdc but not ICA512 $604-979$.

To further compare the serum reactivity against the juxta-membrane (JM) and PTP domain of ICA512 (Fig. 1), autoantibodies to ICA512 ${ }_{604-979}$ were evaluated in competition with excess unlabelled ICA512 $_{1-689}$ or ICA512 ${ }_{687-979}$ (Table 3). Among 86 sera from 122 prediabetic and new-onset patients 
Table 2. Concordance of autoantibodies to ICA512 $2_{604-979}$ and ICA512.bdc in Type I diabetic patients according to varying duration of diabetes

\begin{tabular}{|c|c|c|c|c|c|c|}
\hline & $\begin{array}{l}\text { Prediabetic } \\
\text { and new-onset } \\
(n=122)\end{array}$ & $\begin{array}{l}\text { Recent-onset } \\
<1 \text { year } \\
(n=34)\end{array}$ & $\begin{array}{l}\text { Duration } \\
1-5 \text { years } \\
(n=22)\end{array}$ & $\begin{array}{l}\text { Duration } \\
\text { 5-10 years } \\
(n=30)\end{array}$ & $\begin{array}{l}\text { Duration } \\
10-20 \text { years } \\
(n=35)\end{array}$ & $\begin{array}{l}\text { Duration } \\
>20 \text { years } \\
(n=36)\end{array}$ \\
\hline 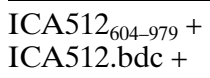 & $74(60.7 \%)$ & $21(61.7 \%)$ & $10(45.5 \%)$ & $17(56.7 \%)$ & $8(22.9 \%)$ & $4(11.1 \%)$ \\
\hline $\begin{array}{l}\text { ICA512 }_{604-979}+ \\
\text { ICA512.bdc - }^{+}\end{array}$ & $12(9.8 \%)$ & $6(17.6 \%)$ & $3(13.6 \%)$ & $3(10.0 \%)$ & $5(14.3 \%)$ & $5(13.9 \%)$ \\
\hline $\begin{array}{l}\text { ICA512 }_{604-979-}- \\
\text { ICA512.bdc + }\end{array}$ & $3(2.5 \%)$ & $2(5.9 \%)$ & $1(4.5 \%)$ & $1(3.3 \%)$ & $3(8.6 \%)$ & $1(2.8 \%)$ \\
\hline $\begin{array}{l}\text { ICA512 }_{604-979}- \\
\text { ICA512.bdc - }^{-}\end{array}$ & $33(27.0 \%)$ & $5(14.7 \%)$ & $8(36.4 \%)$ & $9(30.0 \%)$ & $19(54.3 \%)$ & $26(72.2 \%)$ \\
\hline
\end{tabular}

that reacted positively with ICA512 ${ }_{604-979}, 18.6 \%$ were blocked by ICA512 1 -689 (JM-only epitopes) and $52.3 \%$ with ICA512 $687-979$ (PTP-only epitopes) but the remaining 25 seemed to bear epitopes in both regions. In patients with disease of longer duration, $45 \%$ of sera bound JM epitopes alone and $27 \%$ PTP alone, with the remaining $27 \%$ showing antibodies to both regions. The apparent greater persistence of autoantibodies bound to JM region did not, however, reach statistical significance $(p=0.06)$ (Table 3$)$.

Sequential follow-up of ICA512. We followed 50 subjects of the DAISY cohort from birth. Among siblings or offspring of patients with Type I diabetes (SOC group), 20/40 (50\%) had neither autoantibodies to ICA512 $604-979$ nor ICA512.bdc whereas $15 / 40(37.5 \%)$ had antibodies to both, $4 / 40(10 \%)$ autoantibodies to ICA512 $604-979$ alone and 1/40 (2.5\%) to ICA512.bdc alone. Among a general population cohort (NEC group), seven/ten (70\%) subjects had neither autoantibody specificity, one subject had both, one ICA512 $604-979$, alone and one subject had ICA512.bdc alone. Sequential follow-up of autoantibody titre using ICA512.bdc, ICA512 $604-979$ ICA512 ${ }_{604-979}$, ICA512 ${ }_{689-979}$ and ICA512 ${ }_{1-689}$ constructs did not show consistent differences in the specificities of the autoantibody reactivities during the emergence of disease but did serve to emphasize the importance of the assay sensitivity in early detection. Thus the ICA512.bdc and ICA512 ${ }_{604-979}$ constructs that performed best in terms of the SD score, control mean index and $99^{\text {th }}$ centile index in crosssectional analyses (Table 2) provided the most sensitive index of autoreactivity and its persistence in the four people that were examined.

\section{Discussion}

In this study we show that IA-2/ICA512 molecule is alternatively spliced in the pancreas, brain, thymus, spleen and other neuroendocrine tissues of rodents and humans to a form in which the single transmem-
Table 3. Reactivity of sera measured by antigen pre-absorption analysis using ICA512 $604-979$

\begin{tabular}{llc}
\hline Reactivity & $\begin{array}{l}\text { Prediabetic } \\
\text { and new-onset } \\
(n=122)\end{array}$ & $\begin{array}{l}\text { Duration } \\
>10 \text { years } \\
(n=71)\end{array}$ \\
\hline PTP - only & $45(52.5 \%)$ & $6(27.2 \%)$ \\
JM - only & $16(18.6 \%)$ & $10(45.5 \%)$ \\
Both & $25(29.1 \%)$ & $6(8.4 \%)$ \\
None & 30 & 39 \\
\hline
\end{tabular}

brane domain and some flanking sequence is deleted in frame. Studies of endogenous IA-2 in islet and pituitary have confirmed the existence of multiple molecular forms most of which are attributable to alternative sites of post-translational processing of the molecule. The major form migrates as a doublet of $60,000-65,000 \mathrm{M}_{\mathrm{r}}$ which has a common $\mathrm{NH}_{2}$ sequence suggestive of cleavage by a furin/PC type endopeptidase in the secretory pathway at a dilysine motif $\left(\mathrm{KK}_{448}\right)$. The size variation here is attributed to posttranslational modification or possibly alternative splicing. Other variants are found in bovine pituitary and have been attributed to proteolytic cleavage at sites aa $\mathrm{RR}_{45}, \mathrm{KR}_{133}$ and $\mathrm{KK}_{387}$ in the lumenal domain and $\mathrm{FN}_{644}$ in the cytoplasmic domain respectively.

A protein bearing the aa557-629 deletion of the $\Delta$ exon 13 mutant would be synthesized as a 97,000 $\mathrm{M}_{\mathrm{r}}$ precursor and, if processed at the $\mathrm{KK}_{448}$ site, would generate a $53,000 \mathrm{M}_{\mathrm{r}}$ mature form. Newly synthesized $\Delta$ exon 13 mutant would pass directly into the lumen of the endoplasmic reticulum rather than be inserted into the membrane as in the case of fulllength ICA512. If it folded properly, the mutant would be secreted either constitutively as the 97,000 $\mathrm{M}_{\mathrm{r}}$ form or in a regulated manner as a $53,000 \mathrm{M}_{\mathrm{r}}$ protein assuming that it was processed at $\mathrm{KK}_{448}$ site in a storage granule. It is likely that translocation of the normally "cytoplasmic" domain of $\Delta$ exon 13 into the oxidizing environment of the secretory pathway results in misfolding and proteolysis as part of the post endoplasmic reticulum editing process. Moreover, there are several sites within the "cytoplasmic" do- 
main of ICA512 which would be susceptible to tryptic-like cleavages by the post-translational endoproteolytic machinery of islet cells especially in the aa 629-680 region. The PTP domain itself is insensitive to trypsinolysis both in the native protein [13] and in in vitro translated material [32]. The observation that only lower $\mathrm{M}_{\mathrm{r}}$ forms of soluble ICA512 immunoreactivity were present in human islets would be explicable by such proteolytic events. The $\Delta$ exon 13 mutant mRNA content of islets approximated $30 \%$ of full-length mRNA based upon semiquantitative PCR analysis. It is probable that the relative tissue content of $\Delta$ exon 13 ICA5 512 protein would be less because the full-length protein is delivered efficiently to the granule and retrieved at each round of exocytosis whereas the mutant would be secreted. The finding that around $10 \%$ of the ICA512 in human islets appeared as soluble $\mathrm{NH}_{2}$ terminal immunoreactive forms is consistent with these observations.

The cDNA clone ICA512.bdc which has been used extensively for RIP assays detects antibodies in $60-70 \%$ of prediabetic and new-onset Type I diabetic patients [30]. Most epitopes are confined to the cytoplasmic portion of ICA512 [23, 30, 33] as was confirmed in the present investigation from examination of seven different constructs. These constructs further distinguished between autoreactivity to the conserved PTP domain and the JM aa 604-687 which is unique to ICA512 and phogrin in the PTP superfamily. The latter region is deleted in the ICA512 $\Delta$ exon 13 mutant up to aa 629. In young-onset patients PTP autoantibodies have been shown to be more prevalent and to correspond to $37,000 \mathrm{M}_{\mathrm{r}}$ (phogrin-derived PTP domain) and 40,000 $\mathrm{M}_{\mathrm{r}}$ (ICA512 $654-979$ ) autoantigens defined by trypsin digestion [23]. Juxta-membrane autoantibodies were, however, more prevalent in older-onset patients and found in association with the 40,000 $\mathrm{M}_{\mathrm{r}}$ (ICA512-derived) fragment alone. From our study of new-onset (duration $<7$ days) and long-duration (duration $>10$ years) patients, it seems the JM autoantibodies are initially less prevalent than PTP antibodies (37.7 vs $79.4 \%$ of ICA512 ${ }_{604-979}$ positive subjects) were more persistent ( 72 vs $54 \%$ at $>10$ years duration) which is consistent with these earlier findings.

About $10 \%$ of the reactivity to ICA $512_{604-979}$ was not detected by the RIP assay using ICA512.bdc construct in recent-onset Type I diabetic patients. We also found, however, that about $4 \%$ of Type I diabetic patients at any age or duration, had autoantibodies to ICA512.bdc but not to the ICA512 ${ }_{604-979}$. Although it is conceivable that an epitope upstream of aa557 is present in this construct, it is more likely that the alternatively spliced molecule has additional unique epitopes generated by the joining of exons 12 and 14 on changes in the conformation. From a practical standpoint, it is clear that assays using ICA512 ${ }_{604-979}$ or ICA512.bdc do not simply detect a subset of epitopes exposed on full-length ICA512 and thus assays based on different constructs could yield additional information. Ideally, these should be modelled on the three-dimensional structure of the protein which is still unresolved.

The existence of an alternatively spliced form of ICA512 that shows tissue-specific expression in lymphoid and neuroendocrine tissue raises a number of questions about the way the immune system recognizes this autoantigen in Type I diabetes. The prevailing concept that ICA512 is a secondary target of autoimmunity because the epitopes are cytosolic in location needs to be reconsidered in the light of the finding that the $\Delta$ exon 13 variant is probably secreted in beta cells. The indication that the normally "cytosolic" antigenic domain of the $\Delta$ exon 13 variant enters the secretory pathway could also mean that the molecule can access major histocompatibility complex processing compartments directly and thus be presented to the cellular arm of the immune system. The observation that lymphoid tissues seem to express only the $\Delta$ exon 13 variant could mean that the corresponding sequence is not encountered by the immune system during the process of early thymic education [34] and thus remains a cryptic epitope capable of conferring autoimmunity when expressed peripherally. Such speculations raise readily testable hypotheses that could be addressed using a deletion/mutagenesis strategy similar to that used here for the detection of humoral autoreactivity. Because cellular autoimmunity to the PTP family of proteins has been detected in the non-obese diabetic (NOD) mouse [31], such studies could be extended to an established experimental model of diabetes pathogenesis.

Acknowledgements. This study was supported by Grants DK32083, DK-32493, DK-52068, DK-55597，5-MO1RR00069 (Clinical Research Centers Program) from the National Institutes of Health, Grant Number 1-1998-222 supported by the Juvenile Diabetes Foundation, a grant from the Korea Health 21 R \& D Project, Ministry of Health \& Welfare, Republic of Korea (HMP-00-B-21200-0005), a grant from the Children's Diabetes Foundation at Denver, an American Diabetes Association mentor-based fellowship awarded to K. Kelemen and the JDF Human Islet Distribution Program. M. Solimena, Yale University, is thanked for provision of IA-2 antisera and E. Bonifacio, San Raffaele Scientific Institute, is thanked for provision of the full-length IA-2 cDNA.

\section{References}

1. Greenbaum CJ, Palmer JP, Kuglin B, Kolb H (1992) Insulin autoantibodies measured by radioimmunoassay methodology are more related to insulin-dependent diabetes mellitus than those measured by enzyme-linked immunosorbent assay: results of the Fourth International Workshop on the Standardization of Insulin Autoantibody Measurement. J Clin Endocrinol Metab 74: 1040-1044 
2. Baekkeskov S, Aanstoot HJ, Christgau S et al. (1990) Identification of the $64 \mathrm{~K}$ autoantigen in insulin-dependent diabetes as the GABA-synthesizing enzyme glutamic acid decarboxylase [published erratum appears in Nature 1990; 347: 782]. Nature 347: 151-156

3. Baekkeskov S, Dyrberg T, Lernmark A (1984) Autoantibodies to a 64-kilodalton islet cell protein precede the onset of spontaneous diabetes in the BB rat. Science 224: 1348-1350

4. Daw K, Ujihara N, Atkinson M, Powers AC (1996) Glutamic acid decarboxylase autoantibodies in stiff-man syndrome and insulin-dependent diabetes mellitus exhibit similarities and differences in epitope recognition. J Immunol 156: 818-825

5. Schmidli RS, Colman PG, Bonifacio E (1995) Disease sensitivity and specificity of 52 assays for glutamic acid decarboxylase antibodies. The Second International GADAB Workshop. Diabetes 44: 636-640

6. Chessler SD, Lernmark A (2000) Alternative Splicing of GAD67 Results in the Synthesis of a Third Form of Glutamic-acid Decarboxylase in Human Islets and Other Non-neural Tissues. J Biol Chem 275: 5188-5192

7. Castano L, Russo E, Zhou L, Lipes MA, Eisenbarth GS (1991) Identification and cloning of a granule autoantigen (carboxypeptidase- H) associated with type I diabetes. J Clin Endocrinol Metab 73: 1197-1201

8. Dotta F, Previti M, Lenti L et al. (1995) GM2-1 pancreatic islet ganglioside: identification and characterization of a novel islet-specific molecule. Diabetologia 38: 1117-1121

9. Pietropaolo M, Castano L, Babu S et al. (1993) Islet cell autoantigen $69 \mathrm{kD}$ (ICA69). Molecular cloning and characterization of a novel diabetes-associated autoantigen. J Clin Invest 92: 359-371

10. Rabin DU, Pleasic SM, Shapiro JA et al. (1994) Islet cell antigen 512 is a diabetes-specific islet autoantigen related to protein tyrosine phosphatases. J Immunol 152: 3183-3188

11. Gianani R, Rabin DU, Verge CF et al. (1995) ICA512 autoantibody radioassay. Diabetes 44: 1340-1344

12. Lan MS, Lu J, Goto Y, Notkins AL (1994) Molecular cloning and identification of a receptor-type protein tyrosine phosphatase, IA-2, from human insulinoma. DNA Cell Biol 13: 505-514

13. Payton MA, Hawkes CJ, Christie MR (1995) Relationship of the 37,000- and 40,000-M(r) tryptic fragments of islet antigens in insulin-dependent diabetes to the protein tyrosine phosphatase-like molecule IA-2 (ICA512). J Clin Invest 96: 1506-1511

14. LaGasse J, Jelinek L, Sexson S et al. (1997) An islet-cell protein tyrosine phosphatase is a likely precursor to the $37-\mathrm{kDa}$ autoantigen in type 1 diabetes: human and macaque sequences, tissue distribution, unique and shared epitopes, and predictive autoantibodies. Mol Med 3: 163-173

15. Kawasaki E, Eisenbarth GS, Wasmeier C, Hutton JC (1996) Autoantibodies to protein tyrosine phosphataselike proteins in type I diabetes. Overlapping specificities to phogrin and ICA512/IA-2. Diabetes 45: 1344-1349

16. Lu J, Li Q, Xie H et al. (1996) Identification of a second transmembrane protein tyrosine phosphatase, IA-2beta, as an autoantigen in insulin-dependent diabetes mellitus: precursor of the 37-kDa tryptic fragment. Proc Natl Acad Sci U S A 93: 2307-2311

17. Bingley PJ, Christie MR, Bonifacio E et al. (1994) Combined analysis of autoantibodies improves prediction of IDDM in islet cell antibody-positive relatives. Diabetes 43: $1304-1310$
18. Verge CF, Gianani R, Kawasaki E et al. (1996) Prediction of type I diabetes in first-degree relatives using a combination of insulin, GAD, and ICA512bdc/IA-2 autoantibodies. Diabetes 45: 926-933

19. Bonifacio E, Genovese S, Braghi S et al. (1995) Islet autoantibody markers in IDDM: risk assessment strategies yielding high sensitivity. Diabetologia 38: 816-822

20. Bingley PJ, Bonifacio E, Williams AJ, Genovese S, Bottazzo GF, Gale EA (1997) Prediction of IDDM in the general population: strategies based on combinations of autoantibody markers. Diabetes 46: 1701-1710

21. Rabin DU, Pleasic SM, Palmer-Crocker R, Shapiro JA (1992) Cloning and expression of IDDM-specific human autoantigens. Diabetes 41: 183-186

22. Solimena M, Dirkx R Jr, Hermel JM et al. (1996) ICA 512, an autoantigen of type I diabetes, is an intrinsic membrane protein of neurosecretory granules. EMBO J 15: 2102-2114

23. Lampasona V, Bearzatto M, Genovese S, Bosi E, Ferrari M, Bonifacio E (1996) Autoantibodies in insulin-dependent diabetes recognize distinct cytoplasmic domains of the protein tyrosine phosphatase-like IA-2 autoantigen. J Immunol 157: 2707-2711

24. Naserke HE, Ziegler AG, Lampasona V, Bonifacio E (1998) Early development and spreading of autoantibodies to epitopes of IA-2 and their association with progression to type 1 diabetes. J Immunol 161: 6963-6969

25. Xie J, Zhang B, Lan MS, Notkins AL (1998) Genomic structure and promoter sequence of the insulin-dependent diabetes mellitus autoantigen, IA-2 (PTPRN). Genomics 54: 338-343

26. Rajotte RV, Warnock GL, Coulombe MG (1988) Islet cryopreservation: methods and experimental results in rodent, large mammals and humans. In: Schilfgaarde RV, Hardy MA (eds) Transplantation of the Endocrine Pancreas in Diabetes Mellitus. Elsevier, New York, pp 125-135

27. Ricordi C, Tzakis AG, Carroll PB et al. (1992) Human islet isolation and allotransplantation in 22 consecutive cases. Transplantation 53: 407-414

28. Rewers M, Bugawan TL, Norris JM et al. (1996) Newborn screening for HLA markers associated with IDDM: diabetes autoimmunity study in the young (DAISY). Diabetologia 39: $807-812$

29. American Diabetes Association (1997) Report of the Expert Committee on the Diagnosis and Classification of Diabetes Mellitus. Diabetes Care 20: 1183-1197

30. Kawasaki E, Yu L, Gianani R et al. (1997) Evaluation of islet cell antigen (ICA) 512/IA-2 autoantibody radioassays using overlapping ICA512/IA-2 constructs. J Clin Endocrinol Metab 82: 375-380

31. Kelemen K, Crawford ML, Gill RG, Hutton JC, Wegmann D (1999) Cellular immune response to phogrin in the NOD mouse: cloned T-cells cause destruction of islet transplants. Diabetes 48: 1529-1534

32. Hawkes CJ, Wasmeier C, Christie MR, Hutton JC (1996) Identification of the $37-\mathrm{kDa}$ antigen in IDDM as a tyrosine phosphatase- like protein (phogrin) related to IA-2. Diabetes 45: 1187-1192

33. Bonifacio E, Lampasona V, Genovese S, Ferrari M, Bosi E (1995) Identification of protein tyrosine phosphatase-like IA2 (islet cell antigen 512) as the insulin-dependent diabetes-related $37 / 40 \mathrm{~K}$ autoantigen and a target of islet-cell antibodies. J Immunol 155: 5419-5426

34. Smith KM, Olson DC, Hirose R, Hanahan D (1997) Pancreatic gene expression in rare cells of thymic medulla: evidence for functional contribution to $\mathrm{T}$ cell tolerance. Int Immunol 9: 1355-1365 